\title{
A Virtual Platform to Determine the Tensile Properties of Engineered Cementitious Composite
}

\author{
Suryanto, B. ${ }^{*}$ and Suryanto, J.K. ${ }^{2}$
}

\begin{abstract}
The four-point flexural test is now making headway as an alternative laboratory investigative technique for determining the tensile properties of Engineered Cementitious Composite (ECC) to the more traditional, direct/uniaxial tensile test. As the fundamental mechanics of ECC specimens tested in four-point flexure are well understood, it is possible to develop a simple relationship between flexural test results and the tensile properties of this cement composite. This paper extends this development and aims to provide accessible and quick calculation of the tensile properties of ECC via a virtual test environment. To this end, attention is directed towards the test configurations developed earlier at Heriot-Watt University, the University of Michigan, and Sepuluh Nopember Institute of Technology. In this paper, the technical background employed in creating the virtual environment and the computer implementation using the JavaScript programming language are presented. The prototype virtual environment is freely available via the Internet at https://ecc-calculator.netlify.app/.
\end{abstract}

Keywords: ECC; flexural test; Java Script; online calculator; tensile property; quality control.

\section{Introduction}

Engineered Cementitious Composite (ECC) is a highly ductile family of cement-based materials capable of exhibiting tensile strain capacities several orders of magnitudes higher than ordinary concrete, typically more than $1 \%$ of strain [1]. The most distinctive engineering property of ECC is its strain hardening ability - the ability of the composite to stretch and not fracture under tensile loading, resembling the behaviour of a ductile metal. This is attributed to its ability to form multiple micro-cracks of small widths (typically $<0.1 \mathrm{~mm}$ ) [2-3], rather than a single macro-crack as in ordinary and other types of fibre reinforced concrete [4].

Since the introduction of a specific variant of ECC incorporating Polyvinyl Alcohol fibres in the beginning of the $21^{\text {st }}$ century, ECC has undergone considerable advancements and progressed to the point where it is finding use in a broad range of applications [5-8].

The distinctive engineering properties of ECC may offer economic engineering solutions and therefore have attracted widespread interest from the engineering community. Reasons for which are due to the expectance of ECC to:

1 Institute for Infrastructure and Environment; Heriot-Watt University; Edinburgh; UNITED KINGDOM.

2 Balerno High School; Balerno; Edinburgh EH14 7AQ; Scotland; UNITED KINGDOM.

${ }^{*}$ Corresponding author; Email: B.Suryanto@hw.ac.uk

Note: Discussion is expected before November, $1^{\text {st }} 2020$, and will be published in the "Civil Engineering Dimension", volume 23, number 1, March 2021.

Received 06 May 2020; revised 13 June 2020; accepted 23 June 2020 i. provide a durable repair and cover;

ii. partially replace the steel reinforcement in a heavily reinforced structural element thereby improving constructability;

iii. provide an additional source of energy dissipation and damping effect, whilst offering enhanced structural integrity; and

iv. lower or even eliminate cost of repair in structures subjected to extreme loading conditions such as during seismic events.

From the practical point of view, Kanda and coworkers [2,9] noted the importance of a technically simple test method as part of routine quality control testing to ensure that the tensile properties of ECC in large scale productions are essentially the same as the properties obtained in the pre-qualification stage.

To date, the tensile properties of ECC has been primarily assessed through the direct application of a tensile force at both ends of a test sample, popularly known as the direct/uniaxial tensile test [10-14]. While this test can provide a direct assessment of the tensile properties of ECC, it is difficult to perform to the required standard. This test also requires the use of special testing apparatus and infrastructure, which is often only available in dedicated laboratories, thereby limiting its widespread use in construction. Furthermore, it has been recognised that in most instances, the level of quality required cannot be reached in normal construction practice, resulting in testing carried out exclusively for research purpose in laboratory settings.

Qian and $\mathrm{Li}$ [15] proposed the use of a flexural test to address the limitations of the direct/uniaxial tensile 
test and introduced the concept of master curve. The use of this curve allows for a quick estimation of the tensile strain capacity based on the beam deflection measured at peak load, which is estimated from a linear elastic beam curvature. They further extended their work, albeit using a simplified material model, and developed a series of intermediate calculations to enable the calculation of tensile strength based upon readings from a testing machine [16]. The use of both calculation frameworks would therefore allow tensile strain capacity and tensile strength to be determined.

Suryanto and co-workers [17,18] employed the sectional analysis approach to predict the full loaddeflection response of a test specimen in four-point flexure. In their development, the nonlinear behaviour of ECC and the nonlinear curvature profile due to cracking were fully considered. Furthermore, to promote the uptake of their development among the practitioners, the analytical framework was further implemented in an Excel spreadsheet. They also introduced a series of simple equations to allow quick calculation of the tensile properties of ECC. However, it has been recognised that these equations, and those proposed by Qian and Li [16], are not simple enough for frequent and day-to-day use by technical staff working in material testing laboratories.

In this paper, a user-friendly virtual platform developed using the Hypertext Markup Language/ JavaScript is presented to provide simple and quick calculation of the tensile properties of ECC using fourpoint flexural test results. This virtual page has now been devised and optimised for both desktop and mobile use to promote the use of ECC on a more widespread scale. The first generation of this virtual application can be accessed freely at https://ecccalculator.netlify.app/

\section{Test Setup}

The test configurations adopted in this paper are presented in Figures 1(a)-(c) which include the test configuration used earlier at Heriot-Watt University, with overall specimen dimensions of $20 \times 40 \times 170 \mathrm{~mm}$ [18]; the test configuration currently developed at the Sepuluh Nopember Institute of Technology (as part of the recent mix development [19]) with overall specimen dimensions of $30 \times 75 \times 300 \mathrm{~mm}$; and the configuration used by Qian and $\mathrm{Li}[15,16]$ at the University of Michigan, with overall specimen dimensions of $50.8 \times 76.2 \times 355.6 \mathrm{~mm}$. These test configurations are hereinafter referred to as HWU, ITS and UOM, respectively. All specimens were simply supported and subjected to two equal concentrated loads, with a central constant moment region of $40 \mathrm{~mm}$ for HWU; $90 \mathrm{~mm}$ for ITS and 101.6 $\mathrm{mm}$ for UOM.

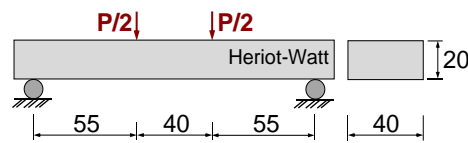

(a)

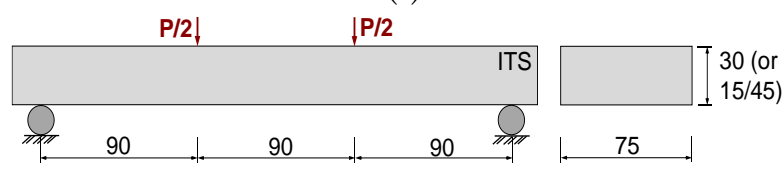

(b)

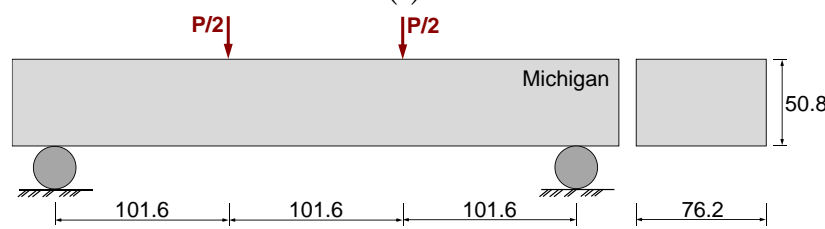

(c)

Figure 1. Test setup: (a) HWU [18]; (b) ITS (c) UOM [15,16]. All dimensions in $\mathrm{mm}$.

\section{Analytical Model: Full Analysis}

\section{Structural Model}

Each specimen shown in Figure 1 is analysed with only half of the length considered due to symmetry. As presented in Figure 2(a), the half model is discretised into 11 segments. At the end of each segment, the cross-section is idealised as a series of thin layers with a width of $b$ and a depth of $d h$ (see Figure 2(b)). The "plane sections remain plane" hypothesis is adopted allowing the ECC strain in each layer $\varepsilon_{\mathrm{i}}$ to be related to the strain at the bottom face, $\varepsilon_{\mathrm{tb}}$, in the following manner [17]:

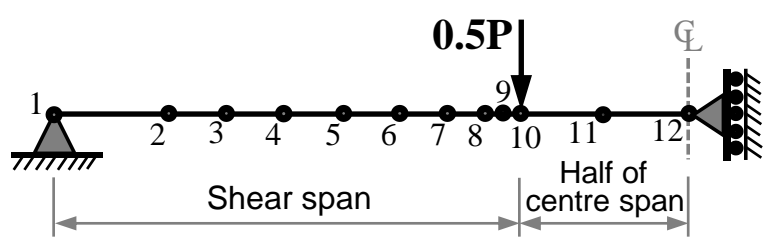

(a)

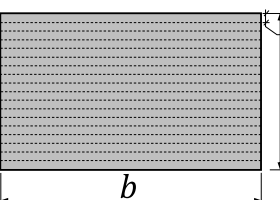

ECC layers

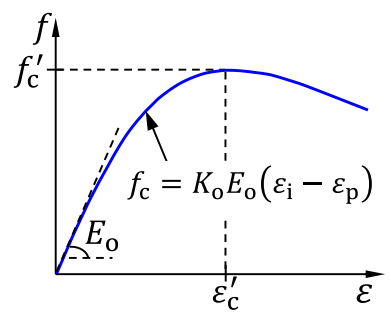

Stress-strain in Compression

(b)

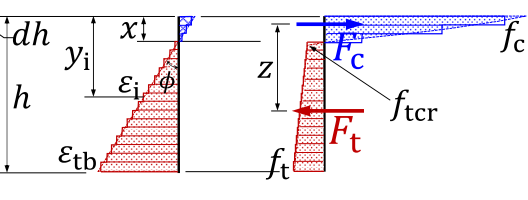

Longitudinal strain Longitudinal stress

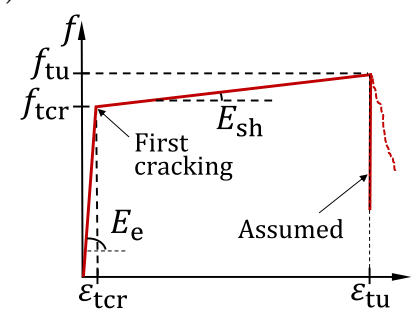

Stress-strain in tension

(c)
Figure 2. (a) Structural Model; (b) Layered Discretisation and Example of Longitudinal Strain and Stress Distributions; (c) Constitutive Relations for ECC [17, 20-22]. 
$\varepsilon_{\mathrm{i}}=\frac{\left(y_{\mathrm{i}}-x\right)}{(h-x-0.5 d h)} \varepsilon_{\mathrm{tb}}$

where $y_{\mathrm{i}}$ is the position of the centroid of layer $i$ from the top of the plate $(\mathrm{mm}) ; x$ is the neutral axis depth from top (mm); $h$ is the overall depth $(\mathrm{mm}) ; d h$ is the layer thickness (mm).

\section{Constitutive Material Models}

The strain in each layer is converted to stress using the constitutive relations presented in Figure 2(c). In each layer, the longitudinal stress and strain are assumed to be uniform and equal to the values at the centre of each layer. The elasto-plastic fracture model [20-22] is used to describe the nonlinear stress-strain response of ECC in compression:

$f_{\mathrm{c}}=K_{0} E_{0}\left(\varepsilon_{\mathrm{i}}-\varepsilon_{\mathrm{p}}\right)$

$K_{0}=\exp \left[-0.73 \frac{\varepsilon_{\mathrm{i}}}{\varepsilon_{c}^{\prime}}\left(1-e^{-1.25 \frac{\varepsilon_{\mathrm{i}}}{\varepsilon_{c}^{\prime}}}\right)\right]$

$E_{0}=2 \frac{f_{c}^{\prime}}{\varepsilon_{c}^{\prime}}$

$\varepsilon_{\mathrm{p}}=\beta\left[\frac{\varepsilon_{\mathrm{i}}}{\varepsilon_{c}^{\prime}}-\frac{20}{7}\left[1-\exp \left(-0.35 \frac{\varepsilon_{\mathrm{i}}}{\varepsilon_{c}^{\prime}}\right)\right]\right] \varepsilon_{c}^{\prime}$

where $K_{0}$ is the fracture parameter; $E_{0}$ is the initial stiffness $(\mathrm{MPa}) ; \varepsilon_{\mathrm{p}}$ is the plastic strain; $f_{\mathrm{c}}^{\prime}$ is the compressive strength $(\mathrm{MPa}) ; \varepsilon_{c}^{\prime}$ is the strain when $f_{\mathrm{c}}$ reaches $f_{\mathrm{c}}^{\prime}$ and can generally be taken as $0.5 \%$; and $\beta$ is the strain rate and taken as 1.0.

The tensile stress of ECC, $f_{\mathrm{t}}$, is related to the initial stiffness $E_{\mathrm{e}}$ and tensile strain, $\varepsilon_{\mathrm{i}}$, [20-22] in the following manner:

$f_{\mathrm{t}}=E_{\mathrm{e}} \varepsilon_{\mathrm{i}}$

After first cracking, the tensile stress in the ECC is assumed to vary linearly with strain at a slope of $E_{\mathrm{sh}}$ during strain hardening, as given by [20]:

$f_{\mathrm{t}}=E_{\mathrm{e}} \varepsilon_{\mathrm{tcr}}+E_{\mathrm{sh}}\left(\varepsilon_{\mathrm{i}}-\varepsilon_{\mathrm{tcr}}\right)$

In the analysis, failure is assumed to take place when the strain at the centroid of the bottom layer $\varepsilon_{\mathrm{tb}}$ at all critical sections (Nodal points 10-12 in Figure 2(a)) reaches the tensile strain capacity of the ECC (i.e. $\varepsilon_{\mathrm{tb}}=\varepsilon_{\mathrm{tu}}$ ). In each analysis step, the resultant forces are checked to ensure equilibrium with the sectional moment applied. If this condition is not satisfied, the strain at bottom fibre is adjusted and the whole analysis is repeated until equilibrium is achieved. Further details of the constitutive relationship, analytical procedure and iteration algorithm are given in reference [17].

\section{Parametric Analysis}

A series of parametric analysis was undertaken on a wide range of possible material properties of ECC: tensile strength in the range 3.3-5.2 $\mathrm{MPa}$; tensile strain capacity in the range $0.5-6 \%$; and compressive strength of 30,45 and $60 \mathrm{MPa}$, respectively.
Figure 3(a) displays the actual values of the material parameters considered, with each analysis case labelled with a number from 1 to 21 . In the first series of analysis (Analysis Cases 1 to 7), the compressive and tensile cracking strengths were set constant at 30 $\mathrm{MPa}$ and 3.2 $\mathrm{MPa}$, respectively. In the second series of analysis (Analysis Cases 8 to 14), the compressive and tensile cracking strengths were taken as $45 \mathrm{MPa}$ and 3.6 MPa, whereas in third series (Analysis Cases 15 to 21), the compressive and tensile cracking strengths were set constant at $60 \mathrm{MPa}$ and $4 \mathrm{MPa}$, respectively. In all cases, the modulus of elasticity was taken as $18 \mathrm{GPa}$.
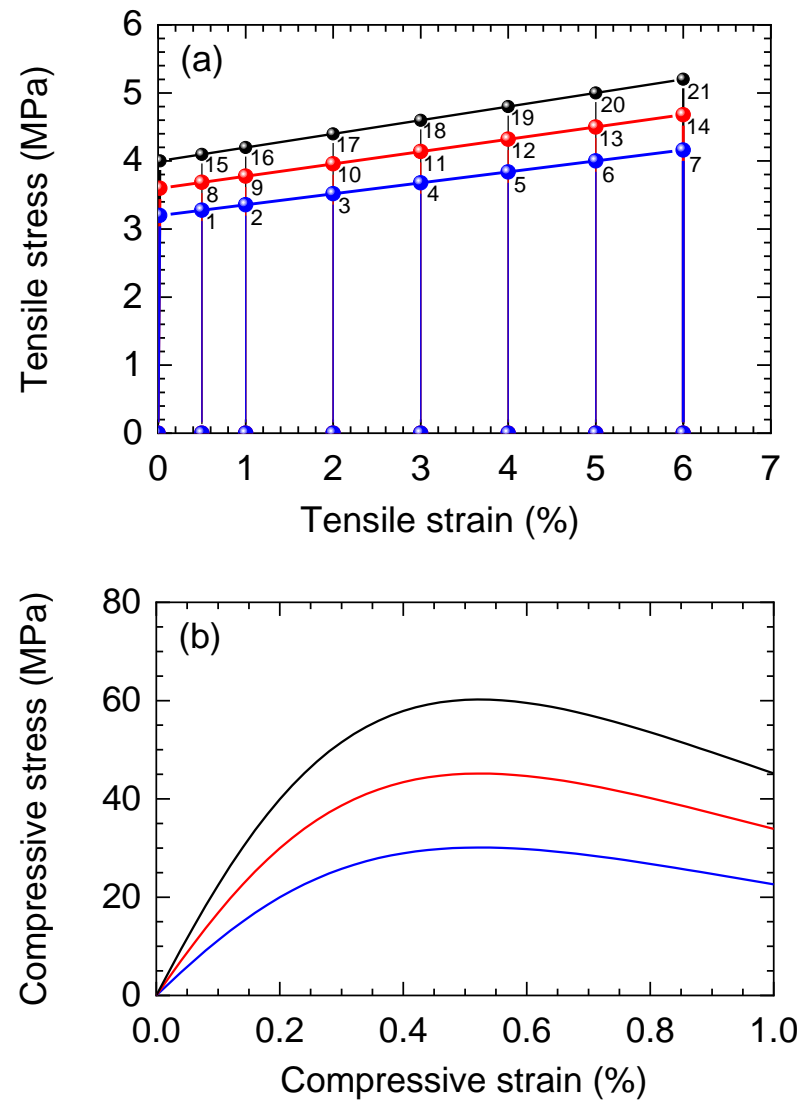

Figure 3. Parameters Considered in this Study: (a) Tensile and (b) Compressive Properties.

\section{Results of Full Analysis}

A typical load-deflection result from the parametric analyses is presented in Figure 4(b) using a tensile property presented in Figure 4(a) and a compressive strength of $45 \mathrm{MPa}$ (Analysis Case 11). The influence of test configuration is clearly evident, with the HWU specimen displaying the smallest load capacity and overall deflection (due to the smallest specimen geometry and span) and the UOM specimen displaying the largest load capacity, but with smaller overall deflection when compared to the ITS specimen. The use of mid-span deflection in place of load-point deflection is recommended as the latter is sensitive to measurement errors. This is addressed in the ITS test configuration. 

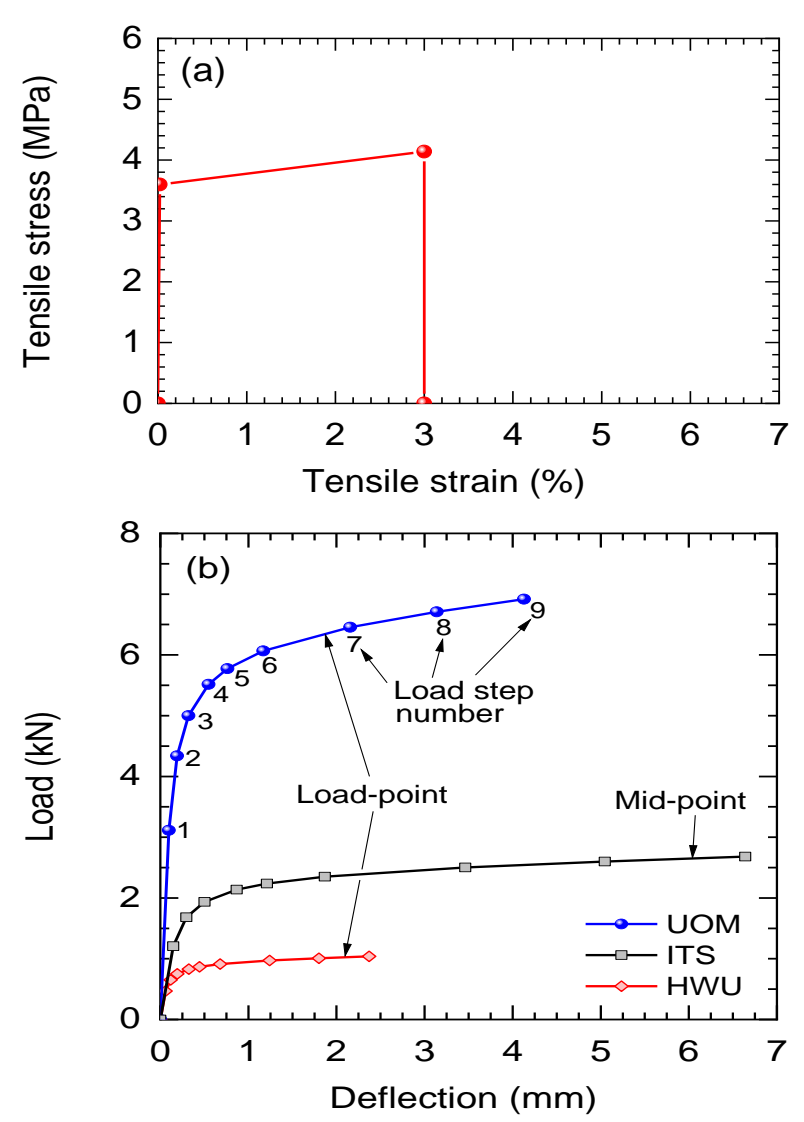

Figure 4. (a) Input Tensile Properties; (b) Predicted LoadDeflection Response for all Test Configurations.

\section{Effect of Tensile Strain Capacity}

Figure 5 presents the predicted load-deflection responses for ITS plate with varying input tensile strain capacities from $0.5 \%$ to $6 \%$ and constant tensile cracking and compressive strengths of $3.6 \mathrm{MPa}$ and 45 MPa (Analysis Cases 8 to 14). It is evident that all these cases produce a similar fundamental loaddeflection response, with all the curves superimposing on each other and with the increase in strain capacity exerting a lengthening effect on the overall response.

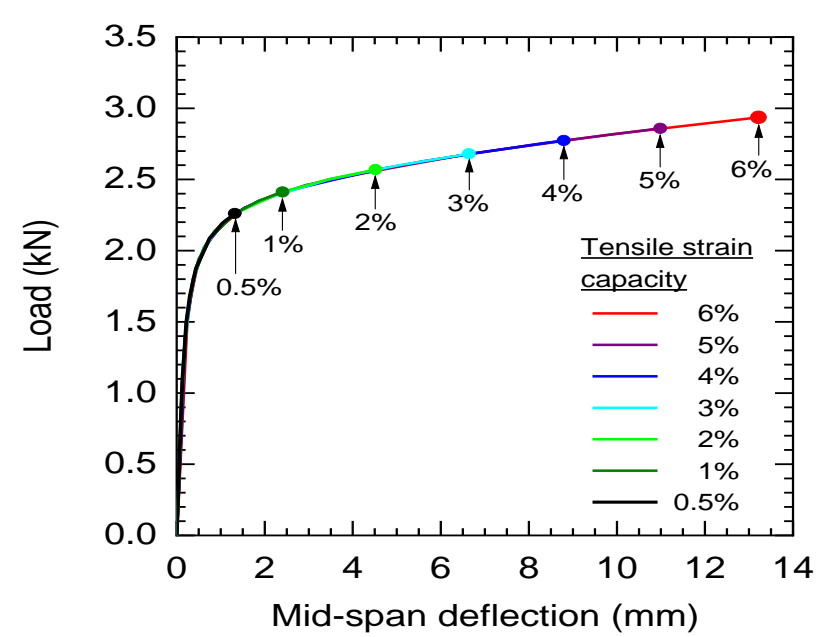

Figure 5. Effect of Tensile Strain Capacity on the LoadDeflection Response of ITS Plate with a Thickness of $30 \mathrm{~mm}$.

\section{Effect of Tensile Strength}

The predicted load-deflection responses for ITS plate with a constant tensile strain capacity of $3 \%$ and varying tensile strengths of $3.68,4.14$ and $4.6 \mathrm{MPa}$ (Analysis Cases 4, 11 and 18) are presented in Figure 6 for illustrative purposes. It is apparent that as tensile strength increases, this results in a progressive increase in load capacity, causing the postcracking response to displace vertically with increasing tensile strength (envisaged as an offset in Figure 6). It is interesting to note that the increase in load capacity is accompanied by a slight reduction in the specimen deflection with the extent of the reduction increasing with increasing deflection.

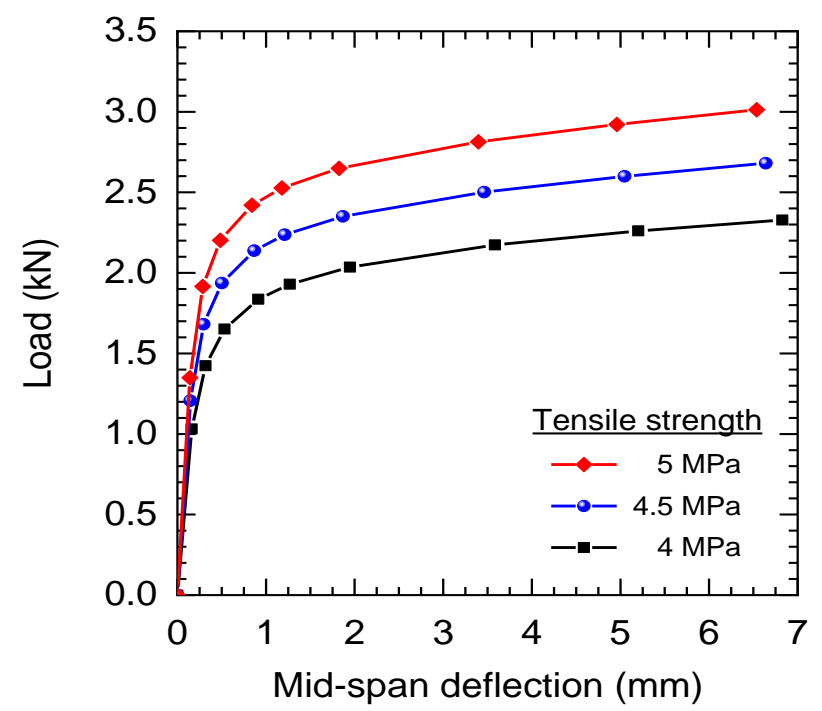

Figure 6. Effect of Tensile Strength on the Load-Deflection Response of ITS Plate with a Thickness of $30 \mathrm{~mm}$. The Tensile Strain Capacity is Taken Constant at 3\%.

\section{Development of Master Curves}

Figure 7(a) presents the relationship between the tensile strain capacity and the mid- or load-point deflections of the specimen at peak load, with the results highlighted with data markers and connected with a dashed line for clarity. It is evident that the tensile strain capacity is proportional to either the mid- or load-point deflection and is virtually independent of tensile and compressive strengths. This implies that the deflection at peak load is the primary controlling influence on the tensile strain capacity, $\varepsilon_{\mathrm{tu}}$. The generic relationships linking these two parameters are

$\begin{array}{llrl}\varepsilon_{\mathrm{tu}} & =1.29 \delta_{\mathrm{l}} n-0.10 & & \text { for HWU } \\ \varepsilon_{\mathrm{tu}} & =0.74 \delta_{1} n-0.10 & & \text { for UOM } \\ \varepsilon_{\mathrm{tu}} & =0.46 \delta_{\mathrm{m}} n-0.10 & & \text { for ITS }\end{array}$

where $\delta_{1}$ and $\delta_{\mathrm{m}}$ are the load- and mid-point deflection at peak load (mm); $n$ is the ratio of the actual thickness to the standard thickness (i.e. $20 \mathrm{~mm}$ for HWU; $50.8 \mathrm{~mm}$ for UOM; and $30 \mathrm{~mm}$ for ITS). These 
equations are plotted in Figure 7(b), so as one can directly read this Figure to determine the tensile strain capacity without the need of performing any calculation.
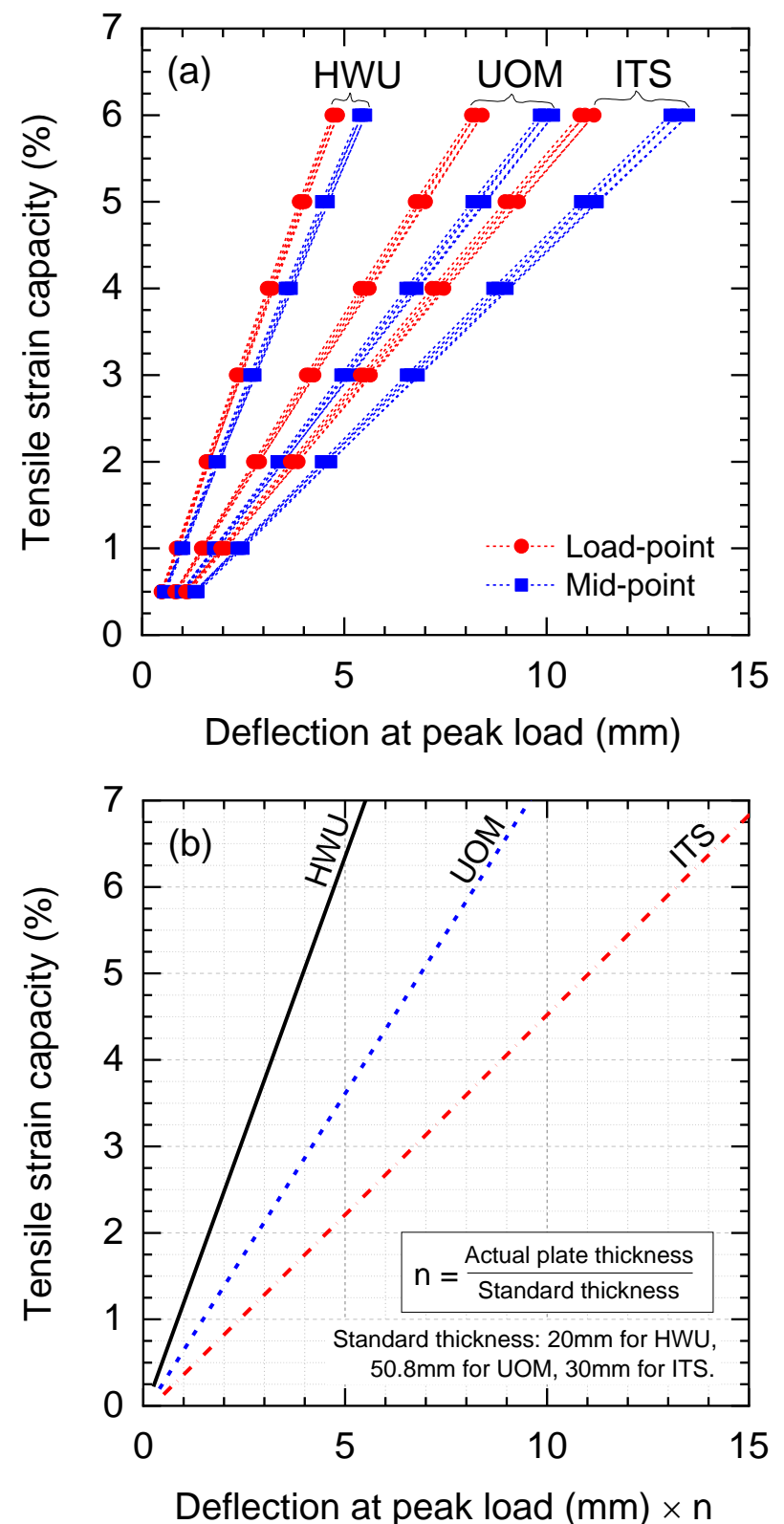

Figure 7. (a) Relationship between Beam Deflection (midand load-point) and Tensile Strain Capacity for the Three Test Configurations; and (b) Proposed Master Curves for Each Setup.

Figures 8(a)-(c) present the relationship between the input tensile strength and the peak load, with the latter multiplied with an empirical constant. It is evident that the tensile strength is linearly proportional to the proposed format of presentation for the peak load. It is interesting to note that although each test configuration is significantly different (and unique of its own) and the response of each plate is highly nonlinear, it is possible to obtain the same linear fit equation.
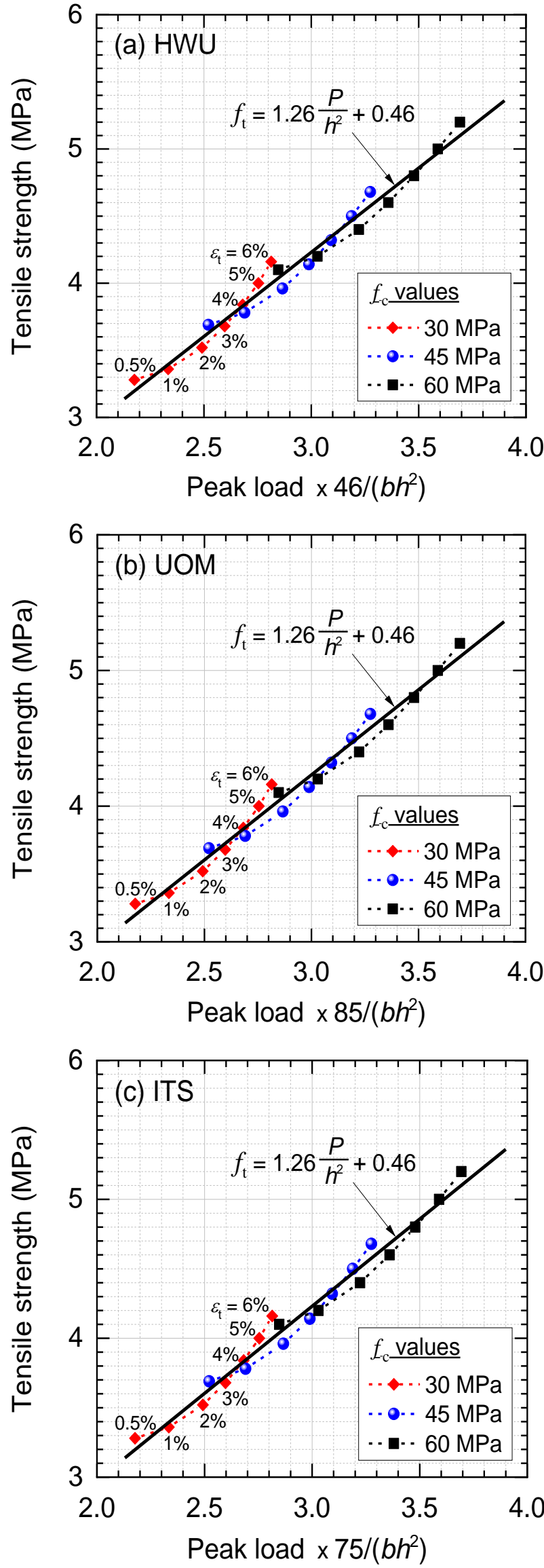

Figure 8. Proposed Master Curve for (a) HWU; (b) UOM; and (c) ITS Specimen. Load in N and Dimensions in $\mathrm{mm}$. 
By presenting the tensile strength and the peak load in the format displayed in Figure 8, a considerably simple expression can be derived:

$f_{\mathrm{tu}}=1.26 \frac{46 P}{b h^{2}}+0.46$ for HWU

$f_{\mathrm{tu}}=1.26 \frac{85 P}{b h^{2}}+0.46$ for $\mathrm{UOM}$

$f_{\mathrm{tu}}=1.26 \frac{75 P}{b h^{2}}+0.46$ for ITS

where $P$ is the peak load $(\mathrm{N}) ; b$ is the overall width $(\mathrm{mm})$ and $h$ is the overall thickness (mm).

\section{JavaScript Implementation}

The above equations have been implemented in an HTML document using the JavaScript programming language. The equations and related diagrams have been generated using the JavaScript to allow the intermediate calculations to be performed in the background based on the user input. Apart from the JavaScript development, the layout and style of the webpage has been designed using the Cascading Style Sheets (CSS) to offer a positive user experience. The developed webpage can be saved from the HTML page to a user local device (i.e. desktop PC, laptop, tablet or mobile phone) and used on a day-to-day basis without internet connection- should access to the internet be limited. If required, a user could also modify the coefficients in the equations to suit the preferred test configuration as they see fit. As feedback is received from the user community, new features will be incorporated to the prototype virtual environment in the future.

Figure 9 presents the developed webpage when opened, displaying two main areas as marked by the light grey and red colours. In the grey (left-hand side) box, the three test configurations are displayed, each preceded by a radio button, and four input boxes for user input. Once a test configuration is selected, the corresponding schematic diagram is displayed in the right-hand side. At the same time, the default values are shown in the input boxes displayed in the lefthand side to indicate the range of reasonable values. Real test data from an actual flexural test can be inputted to replace the default values.

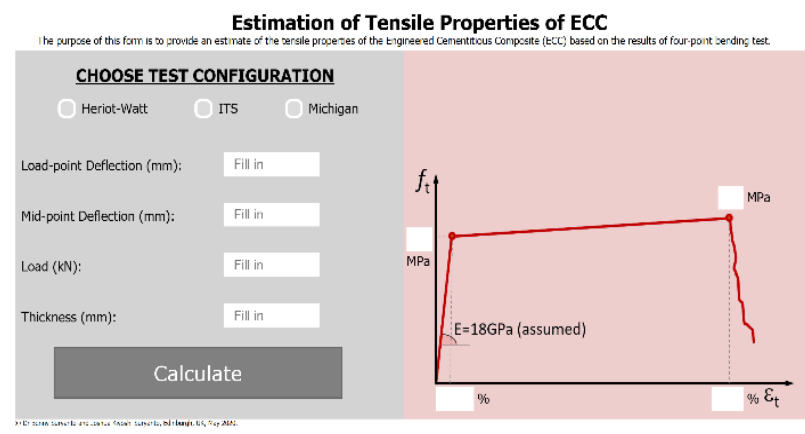

Figure 9. Main Page of the ECC Calculator.

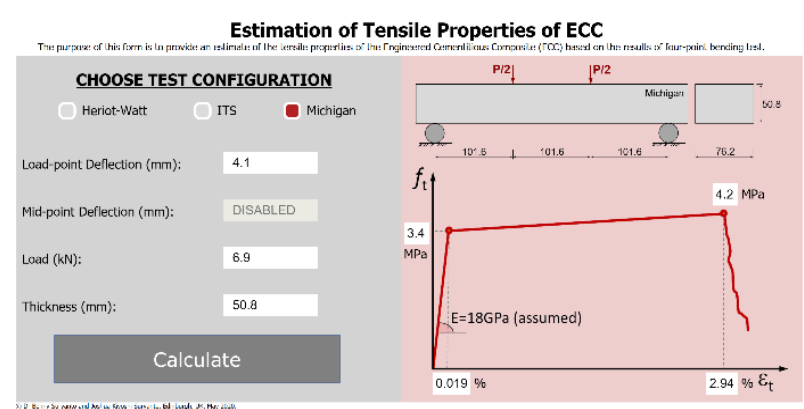

(a)

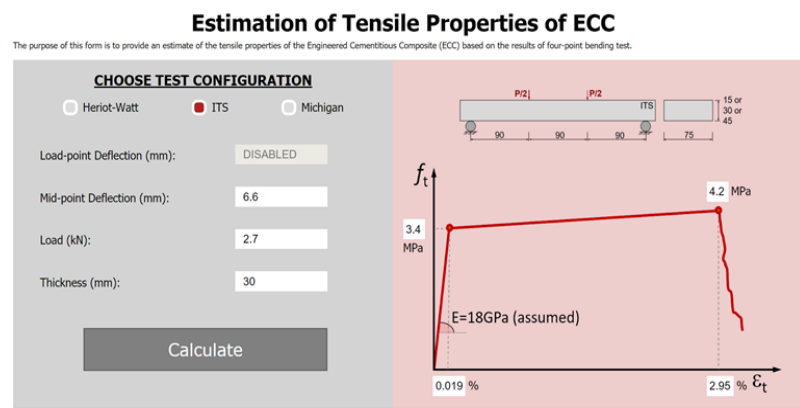

(b)

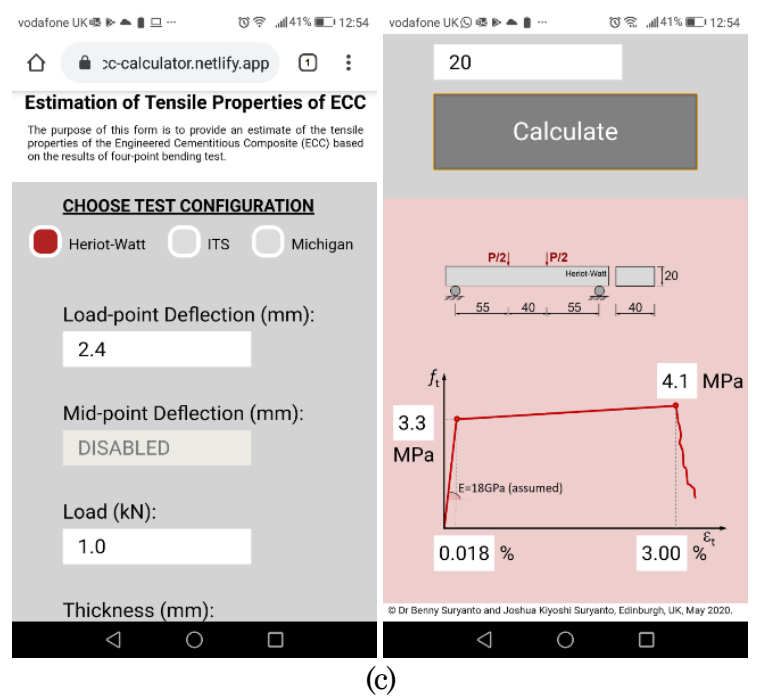

Figure 10. Populated form Displayed in a Laptop with (a) Ultra HD and (b) Full HD Resolutions. (c) the Same form Displayed in a Mobile Phone.

Once the user has provided the necessary input values (or decided to use the default values), the Calculate button can be pressed to trigger the calculations in the background. The computed results are then displayed inside the white boxes displayed around the tensile stress-strain diagram in the righthand side, including the first cracking stress (MPa); tensile strength (MPa); strain at first cracking (\%); and tensile strain capacity (\%).

To maximise the benefits of this development, the webpage has been tailored using Media Queries during the CSS implementation to enable the page to be displayed to various user devices including desktops, laptops, tablets and mobile phones. Figures 10(a) and (b) displays the page layout when opened in 
a device with Ultra HD and Full HD resolutions. In the tablet and mobile versions, the output (light red) box is moved below the input (grey) box due to space limitation, as displayed in Figure 10(c).
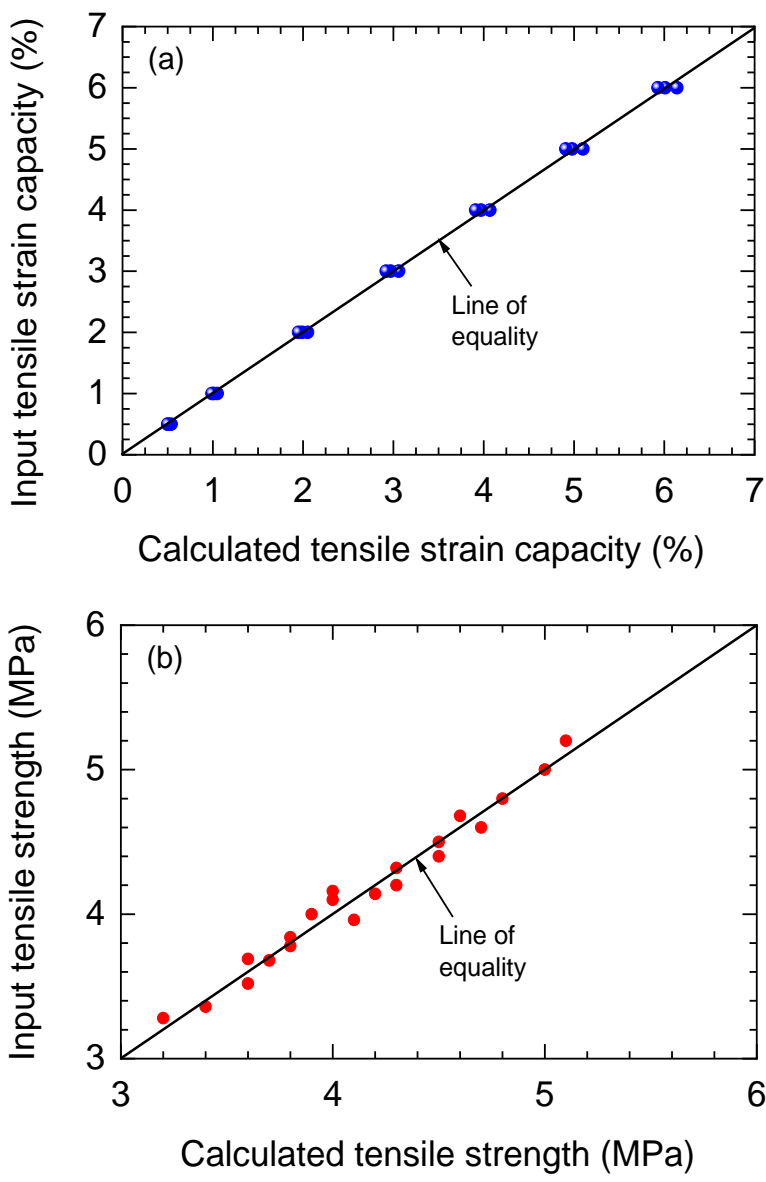

Figure 11. Correlation of Inputted and Calculated Tensile Properties for 21 Analysis Cases.

To check the accuracy of the virtual platform, Figures 11(a) and (b) compare the input tensile strain capacities and tensile strengths from 21 analysis cases to the predicted values obtained from the virtual platform (webpage). For illustrative purposes, results are only presented for the ITS test configuration with a $30 \mathrm{~mm}$ plate thickness. The peak load and deflection values inputted into the webpage were obtained from the full analysis [17]. It was found that the virtual platform produces a mean input-to-predicted tensile strain capacity ratio of 1.01 and a coefficient of variation (COV) of $2.6 \%$, thereby highlighting its similar accuracy as the full analysis. The same level of accuracy is obtained for the tensile strength predictions, with the 21 analysis cases resulting in a mean ratio of 1.0 and a $\mathrm{COV}$ of $2.0 \%$.

\section{Limitations and Future Work}

The virtual platform presented in this paper is introduced to enable rapid estimation of the tensile properties of a cement composite that exhibits tensile strain-hardening behaviour such as the ECC. At this stage, this platform can only be used for the three test configurations introduced earlier, but extensions to other test configurations are very straightforward. For each configuration, it would be possible to use the virtual platform for test specimens of different thicknesses, having due consideration being given to the thickness scaling factor $n$. To this end, it is assumed that the fibre orientation within the composite is random and not affected by specimen geometry (i.e. thickness). In reality, a certain degree of fiber alignment may occur and this is particularly true when the overall thickness of the specimen is less than $\sim 2.5$ times the fibre length. This clearly warrants further investigation and will form the direction of our future work. Further research is also needed to evaluate the accuracy of the platform against experimental data to ensure that the coefficients used in the prediction models are applicable to a wide range of material properties and test parameters. The platform presented in this paper can also be extended for predicting other properties of ECC and different cement-based materials.

\section{Concluding Remarks}

A first generation of virtual platform has been presented and verified against the full analytical framework developed earlier by the first author. Whilst the original framework can predict the full load-deflection response of a prismatic specimen under four-point flexure, it requires the use of an Excel spreadsheet which may not be readily available to technical staff alike. A very simple, user-friendly virtual environment is therefore developed in this work and made freely accessible at https://ecc-calculator.netlify.app/

It has been shown that using the new virtual environment, the tensile strain capacity and tensile strength of ECC can be estimated based on the peak load and deflection obtained from a four-point bending test (i.e. peak load and deflection) with ease, without the need of performing any intermediate calculations. This will have obvious practical impications, with the plaform lending itself to the Civil Engineering and construction materials testing laboratories.

It is envisaged that the virtual platform presented in this paper could, potentially, be further exploited in the following manner:

(1) at the material design stage to examine the effect of modification in mix composition;

(2) in a pre-qualification testing programme to check, for example, the influence of material variability and substitute;

(3) in the development of a new test configuration to advise the range of load and deflection values; 
(4) an educational tool to develop a fuller understanding of the relationship between flexural and tensile properties of ECC;

(5) in a quality control test programme to ensure that the ECC mix, as produced, is substantially the same as the pre-qualified mix; and

(6) as a simple tool to help the development of a standarised test method.

It is yearned that the virtual environment can help in the development of a standarised test method that could one day become as widely well accepted as the cube/cylinder test currently is for ordinary concrete. Finally, it is noteworthy that it is not the intention of the authors to completely replace the direct/unixial tensile test, but rather to accompany it. The virtual test environment could be used alongside with fourpoint flexural test for day-to-day testing, with uniaxial tensile test validating test results periodically.

\section{Acknowledgements}

This work was supported by an Institutional Links grant, ID 414707757, under the Newton Fund Institutional Links Grant and the Ministry of Research, Technology and Higher Education of the Republic of Indonesia partnership. The grant is funded by the UK Department for Business, Energy and Industrial Strategy and the Indonesian Ministry of Research, Technology and Higher Education (Grant No. 5/EI/KP.PTNBH/2019) and delivered by the British Council. The Authors also wish to thank B. Cockburn for his assistance in the early development of the virtual platform, W. Don for proofreading this manuscript and Dr. A. Tambusay for assistance and discussion.

\section{References}

1. Li, V.C., Engineered Cementitious Composites (ECC)-Material, Structural, and Durability Performance, Concrete Construction Engineering Handbook, Nawy E.G. (ed.), CRC Press, pp. 24-1-24-46, 2008.

2. Kanda, T., Tomoe S., Nagai, S., Maruta, M., Kanakubo, T., and Shimizu, K., Full Scale Processing Investigation for ECC Precast Structural Element, Journal of Asian Architecture and Building Engineering, 5(2), 2006, pp. 333-340.

3. Suryanto, B., Wilson, S.A., and McCarter, W.J., Self-healing of Micro-Cracks in Engineered Cementitious Composites, Civil Engineering Dimension, 17(3), 2015, pp.187-194.

4. Suryanto, B., Tambusay, A., and Suprobo, P., Crack Mapping on Shear-critical Reinforced Concrete Beams using an Open-Source Digital Image Correlation Software, Civil Engineering Dimension, 19(2), 2017, pp. 93-98.
5. Fukuyama, H. and Suwada, H., Experimental Response of HPFRCC Dampers for Structural Control, Journal of Advanced Concrete Technology, 1(3), 2003, pp. 317-326.

6. Fukuyama, H., Application of High Performance Fiber Reinforced Cementitious Composites for Damage Mitigation of Building Structures, Journal of Advanced Concrete Technology, 4(1), 2006, pp. 35-44.

7. Kunieda, M. and Rokugo, K., Recent Progress on HPFRCC in Japan, Journal of Advanced Concrete Technology, 4(1), 2006, pp. 19-33.

8. Kanda, T., Nagai, S., Maruta, M., and Yamamoto, Y., New High-rise R/C Structure using ECC Coupling Beams, Proceedings of the $2^{\text {nd }}$ International RILEM Conference on Strain Hardening Cementitious Composites, Rio de Janeiro, Brazil, 2011, pp. 12-14.

9. Kanda, T., Kanakubo, T., Nagai, S., and Maruta, M., Technical Consideration in Producing ECC Pre-cast Structural Element, Proceedings of the International RILEM Workshop on High Performance Fiber Reinforced Cementitious Composites in Structural Applications, 2006, pp. 229-242.

10. Kanakubo, T., Tensile Characteristics Evaluation Method for Ductile Fiber-reinforced Cementitious Composites, Journal of $\mathrm{Ad}$ vanced Concrete Technology, 4(1), 2006, pp. 3-17.

11. Matsumoto, T., Wangsiripaisal, K., Hayashikawa, T., and He, X., Uniaxial TensionCompression Fatigue Behavior and Fiber Bridging Degradation of Strain Hardening Fiber Reinforced Cementitious Composites, International Journal of Fatigue, 32(11), 2010, pp. 1812-1822.

12. Suryanto, B., Wilson, S.A., McCarter, W.J., and Chrisp, T.M., Self-Healing Performance of Engineered Cementitious Composites under Natural Environmental Exposure, Advances in Cement Research, 28(4), 2016, pp. 211-220.

13. Kobayashi, K., Anh, D.L., and Rokugo, K., Effects of Crack Properties and Water-Cement Ratio on the Chloride Proofing Performance of Cracked SHCC Suffering from Chloride Attack, Cement and Concrete Composites, 69, 2016, pp. 18-27.

14. Suryanto, B., Buckman, J.O., Thompson, P., Bolbol, M., and McCarter, W.J., Monitoring Micro-crack Healing in an Engineered Cementitious Composite using the Environmental Scanning Electron Microscope, Materials Characterization, 119, 2016, pp.175185. 
15. Qian, S. and Li, V.C., Simplified Inverse Method for Determining the Tensile Strain Capacity of Strain Hardening Cementitious Composites, Journal of Advanced Concrete Technology, 5(2), 2007, pp. 235-246.

16. Qian, S. and Li, V.C., Simplified Inverse Method for Determining the Tensile Strain Properties of Strain Hardening Cementitious Composites (SHCC), Journal of Advanced Concrete Technology, 6(2), 2008, pp. 353-363.

17. Suryanto, B., Reynaud, R., and Cockburn, B., Sectional Analysis of Engineered Cementitious Composite Beams, Magazine of Concrete Research, 70(22), 2018, pp. 1135-1148.

18. Suryanto, B., Cockburn, B., Han, A.L., and McCarter, W.J., An Alternative Method for Determining Tensile Properties of Engineering Cementitious Composites, Procedia Engineering, 171, 2017, pp. 584-591.
19. Tambusay, A., Suryanto, B., and Suprobo, P., Digital Image Correlation for Cement-based Materials and Structural Concrete Testing, Civil Engineering Dimension, 22(1), 2020, pp. 6-12.

20. Suryanto, B., Mechanics of High Performance Fiber Reinforced Cementitious Composite (HPFRCC) under Principal Stress Rotation, Ph.D Thesis, University of Tokyo, 2009.

21. Suryanto, B., Nagai, K., and Maekawa, K., Modeling ad Analysis of Shear-Critical ECC Members with Anisotropic Stress and Strain Fields, Journal of Advanced Concrete Technology, 8(2), 2010, pp.239-258.

22. Suryanto, B., Nagai, K., and Maekawa, K., 2010, Seared-crack Modeling of R/ECC Membranes incorporating an Explicit Shear Transfer Model, Journal of Advanced Concrete Technology, 8(3), 2020, pp.315-326. 